\title{
JOB BURNOUT IN POLICE DISPATCHERS
}

\author{
Kerry Newness, Nova Southeastern University, U.S.A. \\ Ashley Mutter, Nova Southeastern University, U.S.A. \\ Steven Negron, Nova Southeastern University, U.S.A. \\ Jason Cavich, Nova Southeastern University, U.S.A.
}

dx.doi.org/10.18374/JABE-20-1.1

\begin{abstract}
PurposeJob burnout is an important, multidimensional, and complex construct that we have yet to fully understand. Prior research has shown mixed findings depending upon a number of factors, and we focus on job burnout in the specific occupation of police dispatchers who are civilian employees working in highly stressful occupations and do not receive the same volume of training that law enforcement members do. Thus, understanding the stressors dispatchers encounter and how to mitigate them can provide valuable implications for police departments to consider and possibly implement to improve the health of their dispatchers and effectiveness in the occupation. Method We have survey responses from 45 police dispatchers in the south Florida area assessing the stressors they occur, the possible mitigating factors of social support, communication, and recognition, and how burned out they feel with their occupation. We run hierarchical multiple regression on our variables of interest to distinguish where levels of variance come from in the burnout construct. Results Our results were mixed depending upon the symptom of burnout used as the outcome variable. We found some supporting evidence that role stressors play an important role in leading to job burnout, but not all the time. Being a dispatcher can lead to significant depersonalization from the people they serve, but those effects can be mitigated by having strong social support from their colleagues and supervisors. ConclusionThe results and use of regression analysis with moderating terms provide insights into the dimensions of job burnout that can be controlled with mitigating factors in police dispatch units.
\end{abstract}

Keywords: Job Burnout, Role Stressors, Organizational Culture, Police Dispatchers 